\title{
Análisis según el Modelo Cognitivo APOS* del Aprendizaje Construido del Concepto de la Derivada
}

\author{
Analysis According to the Cognitive Model Following Constructed \\ Learning of the Concept of Derivative
}

\author{
M. Angélica Vega Urquieta** \\ José Carrillo Yañez ${ }^{* * *}$ \\ Jorge Soto Andrade ${ }^{* * * * *}$
}

\begin{abstract}
Resumen
Este trabajo fue motivado por el bajo rendimiento de los estudiantes que ingresan a primer ciclo en la educación superior en Chile. Por lo tanto, nuestro interés fue investigar cómo aprenden matemáticas los estudiantes, cómo construyen conocimiento y cuál es el nivel de aprendizaje construido en un tema, de especial dificultad para ellos, como es el concepto de la derivada y sus aplicaciones. Este trabajo es parte de una investigación más extensa que consideró un estudio de casos múltiples. Presentamos, aquí, los resultados obtenidos de dos de ellos. Para indagar el aprendizaje construido se usó el modelo cognitivo APOS de Ed Dubinsky. Se creó un entorno interactivo de enseñanza para el aula apoyado por Módulos de Clases y clases de trabajo práctico basado en Talleres Computacionales. Se mencionarán algunos resultados brevemente. Se descubrió que, si el concepto de derivada en un punto no es comprendido a nivel de Acción, los estudiantes tienen dificultades para extenderlo y transitar a un nivel superior de comprensión e interpretar el concepto geométricamente. Además, tienen dificultades de comprensión cuando deben discriminar entre proposiciones falsas y verdaderas enunciadas sobre propiedades de la derivada relacionadas con la monotonía y la concavidad de una función. Se obtuvieron conclusiones sobre el aprendizaje evidenciado y sobre la estrategia de enseñanza implementada.
\end{abstract}

Palabras-clave: Modelo Cognitivo APOS. Descomposición Genética de un Concepto. Derivada. Estudio de Casos.

\begin{abstract}
This work was motivated by the poor performance of first year undergraduates in college. Therefore, our interest was to investigate how students learn mathematics, how they construct knowledge, and which is the level of their constructed learning of a mathematical content that is especially difficult for them, i.e. the concept of derivative and its applications. This work is part of a broader research that considered a multiple case study. Here we present the results obtained from two of them. To investigate constructed learning we used Dubinsky's APOS cognitive model. We created an interactive teaching environment, supported by classroom lessons and class modules of practical work based on Computer Workshops. Some results will be mentioned briefly. It was found that if the concept of "Derivative at a point" is not understood at the level of action, the students have difficulty

\footnotetext{
* Action Process Object Schema

${ }^{* *}$ Doctora en Educación Matemática por la Universidad de Huelva. Profesor Asociado en la UCHILE, Santiago, Chile. Dirección Postal: Sergio Livingston 1007, Comuna de Independencia, 8380000, Santiago, Chile. E-mail: mavega@ciq.uchile.cl

${ }^{* * *}$ Doctor en Educación Matemática por la Universidad de Huelva. Catedrático de universidad del Área de Didáctica de la Matemática en la UHUELVA, Huelva, España. Dirección Postal: Avda. 3 de Marzo s/n, E21071, Huelva, España. E-mail: carrillo@uhu.es

${ }^{* * * *}$ Doctor en Ciencias Matemáticas por la Universidad de Paris 11. Profesor Titular en la UCHILE, Santiago, Chile. Dirección Postal: Las Palmeras 354, Ñuñoa, Santiago, Chile. E-mail: sotoandrade@u.uchile.cl
} 
in extending it and moving to a higher level of understanding and interpreting the concept geometrically. They also have difficulties understanding when they have to discriminate between true and false propositions set out on properties of the derivative related to monotony and concavity of a function. Conclusions were drawn on learning achieved and on the teaching strategies being implemented.

Keywords: APOS Cognitive Model. Genetic Decomposition. Derivative. Case Studies.

\section{Introducción}

La enseñanza tradicional ${ }^{1}$, en el sentido dado en Barbosa (2003, p. 2), utilizada en la enseñanza de la Matemática, no ha solucionado el problema general de rendimiento y desmotivación de los estudiantes por las asignaturas del área Matemática. Por lo tanto, se considera de suma importancia desarrollar acciones orientadas a incentivar el estudio de la Matemática, usando recursos adecuados y actualizados, así como psicopedagógicamente válidos, de modo de interesar a los estudiantes y accionar procesos que produzcan un aprendizaje significativo.

El propósito de este trabajo es indagar el aprendizaje construido del concepto de la derivada y algunas de sus aplicaciones. En la sociedad actual el ordenador forma parte del entorno cotidiano, por tanto, en el transcurso de esta investigación se ha introducido un entorno de trabajo que sea familiar y estimulante para que los estudiantes participen activamente en su aprendizaje, implementando como parte de la estrategia de enseñanza el uso de la herramienta tecnológica. Ideas compartidas, también, por los investigadores Codes y Sierra (2005). La intención es proporcionar entornos de interés, favoreciendo las condiciones necesarias para que los estudiantes desarrollen distintos registros: analítico, geométrico y gráfico. Parte de la inquietud del estudio original fue indagar respecto de:

1. ¿Qué procesos cognitivos se producen al relacionar el concepto de función derivada con otros conceptos? (propiedades cualitativas de una función y modelación y resolución de algunos problemas mediante una ecuación diferencial)

2. ¿Cuál es el nivel de aprendizaje construido por los estudiantes de una muestra?

En este artículo, solo analizamos y discutimos los resultados relacionados con estas preguntas, usando el modelo cognitivo APOS de Ed Dubinsky para analizar el desarrollo de la comprensión. Otras interrogantes, que forman parte de un estudio más amplio, se incluyen en Vega (2012).

\footnotetext{
${ }^{1}$ Sistema pedagógico que no se fundamenta en una investigación científica en Educación Matemática y que, en general, no ha logrado avances en el aprendizaje de los conceptos matemáticos.
} 
El presente estudio se organizó en cinco secciones. En la primera, se presenta los antecedentes generales que han motivado el trabajo, así como el desafío de querer mejorar la práctica educativa, que se traduce en indagar y obtener evidencias del aprendizaje construido por las estudiantes y responder las interrogantes que surgen en forma natural ante la problemática planteada. La segunda sección es el marco teórico, en el que se presentan las correspondientes bases teóricas necesarias en la que se sustenta esta investigación, a saber, el modelo cognitivo APOS de Dubinsky (1996). La tercera sección es el diseño de la investigación que hemos subdivido, a su vez, en 5 sub-secciones. En la primera se presenta el problema de investigación y los objetivos; en la segunda se caracteriza esta investigación y la selección de los casos. La tercera comprende la descripción de la estrategia de enseñanza utilizada; en la cuarta presentamos los instrumentos de obtención de la información y por último, en la quinta sub-sección describimos el instrumento de análisis. La cuarta sección corresponde al análisis y discusión de los resultados obtenidos y, finalmente, en la quinta sección se exponen las conclusiones de este estudio.

\section{Marco teórico}

Varios son los trabajos y autores que se podrían citar como fundamento de este trabajo en relación del modelo cognitivo utilizado. Sin embargo, se destaca el hecho de que los trabajos de Piaget (1975) son, en cierta forma, la base para algunas investigaciones posteriores, como las de Dubinsky (1991), Tall y Vinner (1981), Sfard (1994) y los aportes de Clark et al. (1997), que contribuyeron a mejorar la fase de esquema de la teoría de Dubinsky, basándose en la tríada de Piaget y García (1983). Estos trabajos son un gran aporte al mejoramiento de la enseñanza y aprendizaje de las matemáticas, por tanto un valioso material para esta investigación. Hemos escogido el modelo cognitivo APOS puesto que la estrategia de enseñanza usada por Asiala et al. (1996) se asemeja a la de esta investigación.

La teoría APOS (Acción-Proceso-Objeto-Esquema) surge como un intento de entender el mecanismo de la abstracción reflexiva introducida por Beth y Piaget $(1980)^{2}$ y que constituye la noción central de la teoría de Piaget sobre la construcción del conocimiento por los individuos. Según Dubinsky (1991, p. 1):

\footnotetext{
el concepto de abstracción reflexiva puede ser una poderosa herramienta en el estudio del pensamiento matemático avanzado, que puede proporcionar una base teórica que apoye y contribuya a nuestra comprensión de qué es y cómo podemos ayudar a los estudiantes a desarrollar la capacidad de participar en ella.
}

\footnotetext{
${ }^{2}$ Piaget llama así a la abstracción que parte de las acciones u operaciones y no meramente de los objetos.
} 
Para Dubinsky (1991) y su equipo, la abstracción reflexiva será la construcción de objetos mentales y de acciones mentales sobre esos objetos. Definen el concepto de esquema como una colección más o menos coherente de objetos y procesos. Por el término objeto se refieren a un objeto mental o físico, y por proceso (o proceso mental) a una acción mental de naturaleza interna del individuo. Puntualizan que uno de los propósitos de elaborar una teoría general es aislar pequeñas porciones de esta estructura compleja y dar descripciones explícitas de posibles relaciones entre esquemas. Afirman que, no es fácil separar una descripción del conocimiento matemático de su construcción, y que no es posible observar directamente los esquemas de un individuo o sus objetos y procesos. Solo se puede inferir de las observaciones que un individuo puede o no enfrentar problemas o situaciones en las que está buscando una solución o tratando de comprender un fenómeno. Dubinsky (1991) plantea la idea de usar experiencias computacionales para ayudar a los estudiantes a crear abstracción reflexiva, como una forma de lidiar con los obstáculos epistemológicos y conflictos.

En la teoría APOS el desarrollo de la comprensión comienza con la manipulación de objetos físicos o mentales previamente construidos para formar acciones, las acciones se interiorizan para formar procesos los que a su vez se encapsulan para formar objetos (hace notar, sin embargo, que en muchas operaciones matemáticas es necesario desencapsular un objeto y trabajar con el proceso del cual proviene) y finalmente las acciones, los procesos y los objetos se pueden organizar en esquemas. APOS es una teoría constructivista que comienza con la hipótesis:

[...] el conocimiento matemático de un individuo es su tendencia ${ }^{3}$ para responder ante las situaciones matemáticas problemáticas, reflexionando sobre ellas dentro de un contexto social mediante la construcción o reconstrucción de acciones, procesos y objetos matemáticos, y organizándolos en esquemas puede ser capaz de dar solución a estas situaciones (ASIALA et al., 1996, p. 5).

Luego, cada sujeto tendrá una serie de esquemas. Por lo tanto, un esquema no puede ser construido sin el prerrequisito de esquemas existentes. Esto es, a menudo, ignorado en la enseñanza tradicional.

\footnotetext{
${ }^{3}$ Para Asiala et al. (1996), el término tendencia se refiere a las relaciones entre la construcción mental y las interconexiones que un individuo utiliza para entender un concepto y la forma en que el individuo las usa (o falla en el uso) en situaciones problemáticas.
} 


\section{Diseño de la investigación}

\subsection{El problema de investigación y objetivos}

El problema del bajo rendimiento de los estudiantes que ingresan a las universidades chilenas es antiguo. Es sabido que las asignaturas de matemáticas crean dificultades en el aprendizaje en la mayoría de ellos. Los factores son variados, desde cursos masivos, problemas que traen consigo desde la Enseñanza Media, que se traducen en vacíos en las materias, inmadurez emocional y el salto cuántico, que representa la gran diferencia del paso de Enseñanza Media a Enseñanza Universitaria, entre otros. Una forma de acortar esta gran brecha es mejorar la práctica educativa, incorporando estrategias de enseñanza que faciliten un rol participativo en lugar del habitual rol pasivo desarrollado en el estudiante, apoyada de recursos tecnológicos que para los adolescentes resulta motivador.

Reflexionando sobre estas ideas surgen algunas preguntas: ¿Cómo modificar la práctica educativa con el propósito de mejorar el rendimiento de los estudiantes en matemáticas?, específicamente en uno de los temas de difícil comprensión para ellos, como es la derivada y algunas de sus aplicaciones. Un supuesto implícito en esta pregunta es el nexo entre modificaciones de la práctica educativa y elevar el rendimiento estudiantil con las construcciones mentales que debe realizar un estudiante para comprender estos conceptos matemáticos. Por lo tanto, se plantea el problema de investigar: cuál es el nivel de aprendizaje construido de una muestra de estudiantes que cursan la asignatura de Matemáticas I de la carrera de Ingeniería en Alimentos. Este estudio se realizó desde la perspectiva del modelo cognitivo APOS, al utilizar nuevas estrategias de enseñanza.

En este artículo se analizan dos de los cuatro casos de la muestra para no extender este análisis. El interés es obtener evidencia del aprendizaje construido por cada estudiante de acuerdo al modelo APOS.

\subsection{Caracterización y casos}

Se realiza un estudio de casos, es decir, un método de investigación cualitativa que se está utilizando ampliamente para conocer la realidad social y educativa. Según Stake (1998, p. 9): "es el estudio de la particularidad y complejidad de un caso singular para llegar a comprender su actividad en circunstancias importantes”. La selección de los casos se realizó considerando el nivel de los conocimientos mínimos que deberían tener los estudiantes al 
ingresar a la universidad. Se usó como referente una Prueba de Diagnóstico, que se aplicó a cada estudiante al inicio del curso. El criterio utilizado fue seleccionar estudiantes con calificaciones bajas y altas en forma aleatoria ${ }^{4}$. Posteriormente, se ratificaron los casos según los resultados obtenidos en una segunda prueba diagnóstica, después de un mes de reforzamiento intensivo de los conocimientos mínimos obligatorios. Con el fin de mantener la confidencialidad tanto de las encuestas aplicadas, como de las pruebas se consideró oportuno asignar a los estudiantes nombres ficticios. En este caso serán: Daniela y Lola.

Daniela obtuvo nota (1.5) en la primera prueba, luego del reforzamiento logró subir su calificación a (5.0) en la segunda prueba diagnóstica. Lola obtuvo (6.5) en la primera prueba y después del reforzamiento bajo su calificación en un punto a (5.5). Estos resultados indican que Daniela muestra un progreso y que Lola conoce los temas exigidos como mínimos. En ambos casos, es interesante realizar un seguimiento para conocer cómo evoluciona y se relaciona el aprendizaje construido con los nuevos conceptos. Como parte de la estrategia de aprendizaje se implementó:

\section{Módulos de Clases ${ }^{5}$}

2. Laboratorios Computacionales ${ }^{6}$, usando el software MAPLE 8. (Adquirido con licencia de campo para varias facultades de la universidad.)

3. La interacción social entre todos los actores del proceso-enseñanza.

\subsection{Estrategia de enseñanza}

Se desarrolla un modelo similar al comentado por Asiala et al. (1996), es decir, se incluye un análisis teórico, diseño instruccional, y observaciones y evaluaciones. El análisis teórico se basa en el modelo cognitivo denominado por Dubinsky (1991) descomposición genética. En esta investigación se realizó la descomposición genética del concepto en estudio, de los módulos de clases y de los laboratorios computacionales. Las observaciones y evaluaciones fueron realizadas a partir de los datos recogidos mediante los instrumentos secundarios y el instrumento de análisis. Se denominaron instrumentos secundarios a aquellos usados para evaluar el rendimiento de los estudiantes durante el curso de Matemáticas I, es decir, son las evaluaciones planificadas regularmente que forman parte del curriculum

\footnotetext{
${ }^{4}$ La escala de calificaciones utilizada es la definida por el Ministerio de Educación (MINEDUC): 1.0 (Malo), 2.0 (Deficiente), 3.0 (Menos que suficiente), 4.0 (Suficiente), 5.0 (Bueno), 6.0 (Muy bueno) y 7.0 (Sobresaliente).

${ }^{5}$ Pequeño apunte con el enfoque particular dado en esta asignatura. Incluye contenidos teóricos con actividades grupales para desarrollar dentro y fuera del aula.

${ }^{6}$ El laboratorio computacional es una clase que se realiza en un aula interactiva, siguiendo una pauta con instrucciones y problemas modelos ilustrativos.
} 
diseñado para cada carrera. El instrumento de análisis no forma parte de las evaluaciones de la asignatura y es el que permite analizar el aprendizaje construido por cada estudiante del concepto en estudio. En la sección Metodología de la Enseñanza se describe la descomposición que se propone para el concepto, los módulos de clases y los laboratorios computacionales.

En general, una descomposición genética de un concepto se define como el análisis teórico del concepto en función de las construcciones mentales que un estudiante debería realizar para desarrollar la comprensión de éste. (ASIALA et al., 1996)

\subsubsection{Metodología de la enseñanza}

El mejorar la práctica social y/o educativa debería producir un cambio de actitud en el estudiante, y, luego, en el aprendizaje construido que es el objetivo en este estudio. Bajo esta hipótesis, para estimular el aprendizaje de la función derivada, uno de los aspectos fundamentales fue crear entornos interactivos de enseñanza, tanto en el aula como para el trabajo práctico consistente en los laboratorios computacionales. Se realizaron las siguientes actividades:

1. A cada estudiante se le entregó un módulo de clases cada 15 días aproximadamente. En total se entregaron 7 módulos en función de la extensión de los contenidos a tratar. Los módulos contienen los temas más relevantes a discutir en el aula. Adicionalmente, este material se divulgó a través de la página web confeccionada por la profesora para este curso. El semestre consta de 15 semanas, la asignatura de 6 horas a la semana, que comprenden tanto las clases teóricas como aquellas en aula interactiva, en total 90 horas semestrales.

2. A cada estudiante se le asignó un PC en la sala de laboratorio computacional.

3. A cada estudiante se le entregó por escrito un pequeño manual MAPLE con las instrucciones básicas que permiten utilizar el software sin dificultad.

El trabajo en el aula se basó en la discusión de los conceptos tratados en los módulos de clases, que contenían definiciones, teoremas, problemas ilustrativos y actividades para desarrollar en la sala de clases de forma grupal. La dinámica de la clase se basó en formar grupos por afinidad e iniciar la discusión de los contenidos. Se escoge un estudiante al azar, que actúe como monitor, que inicie el diálogo en voz alta de los conceptos para generar el análisis y discusión en el aula. Posteriormente, el profesor hace preguntas para percibir si se comprendió la notación, significado y la relación con algún conocimiento previo (acción). Si existieran dificultades en la comprensión de algún concepto, notación o demostración el 
profesor debe dilucidar las dudas en la pizarra y asegurar que todos los estudiantes comprendan el tema. Los alumnos discuten y resuelven problemas en conjunto y, finalmente, realizan las actividades planteadas en grupos, de modo de favorecer el trabajo colaborativo y la sociabilización entre ellos. Es decir, se pretende mediante la repetición de la acción inducir al estudiante para que desarrolle la etapa de proceso. En sesiones posteriores, cuando los nuevos conceptos hayan sido internalizados, se lleva a los estudiantes al laboratorio computacional (aula interactiva) para reactivar las acciones cognitivas desde otra dimensión, que agrega, además, la visualización gráfica.

El laboratorio constituye un nuevo estímulo para repetir el ciclo (A-P-O) y utilizar las propiedades inherentes al objeto para realizar nuevos manejos a partir de él y facilitar la asimilación del concepto. Las actividades que deben realizar los estudiantes se exhiben mediante un proyector. Entre ellas se incluyen las instrucciones necesarias para abordar con éxito las actividades que reforzarán el aprendizaje de los conocimientos discutidos en el aula.

El taller práctico tiene varios propósitos, entre ellos anclar conocimientos a través de la visualización, desarrollar las facultades de comprensión y creatividad en los estudiantes, reforzar los conceptos discutidos, realizar un nuevo estímulo que reactive su red neuronal de la memoria y así obtener un mejor aprendizaje ${ }^{7}$. La participación del profesor consiste en aclarar, explicar, dar las instrucciones y comandos propios del software para una mejor comprensión. El trabajo que desarrollan los estudiantes en el aula les permitirá familiarizarse con las sentencias, instrucciones y bibliotecas matemáticas que usarán en las clases sucesivas y en los talleres siguientes. Por tanto, se les permite conservar la hoja de cálculo (worksheet).

Cada dos laboratorios aproximadamente, se realizó un control, cuyo propósito fue validar lo aprendido. Los estudiantes desarrollaron una pauta en forma individual con evaluación que, al término de la sesión, se envió por correo electrónico al ayudante o al profesor del curso para su corrección. Adicionalmente, se les entregó un laboratorio grupal, dos estudiantes por grupo, con evaluación, cuyo objetivo fue producir interacción y generar trabajos colaborativos. Se realizaron 6 sesiones prácticas y 2 evaluaciones individuales. Se analiza, a continuación, como se relaciona el modelo cognitivo APOS con el material didáctico elaborado y modus operandi.

\footnotetext{
${ }^{7}$ Ausubel (1978) lo denomina aprendizaje significativo.
} 


\subsubsection{Descomposición Genética Inicial del Concepto}

En todo proceso de aprendizaje interesa que los estudiantes logren idealmente la estructura cognitiva de la fase superior, es decir, un esquema sub-etapa Trans, que, mediante acciones, procesos, objetos y otros esquemas, el estudiante pueda evocar para resolver un determinado problema. Para ello el estudiante debe haber logrado primero el esquema de una función y sus propiedades, las operaciones entre funciones, la composición y descomposición de funciones, así como el esquema sobre la comprensión del límite de una función. Asimismo, la descomposición genética del concepto de límite de una función fue realizada por Aneshkumar (2010), y el de la regla de la cadena por Clark et al. (1997). Con estos esquemas en mente, se realiza una descomposición genética del concepto derivada.

1. Para desarrollar la comprensión a nivel de acción, el estudiante debe entender el concepto de derivada en un punto; su interpretación geométrica como una aproximación a la pendiente de la recta tangente en un punto de la gráfica de f, que se obtiene como el límite de una sucesión de pendientes de rectas secantes cuando la longitud del intervalo de base tiende a cero; la interpretación física de la velocidad instantánea que se obtiene por el paso al límite de una sucesión de velocidades medias sobre intervalos cuya longitud se aproxima a cero; calcular derivadas en un punto usando límites de funciones elementales como polinómicas, raíces, inversas proporcionales etc.

2. A nivel de proceso se consideró el manejo de la operatoria y demostraciones simples de las propiedades de la función derivada, como determinar fundamentando la derivada de una potencia de grado $\mathrm{n}$, derivada de una suma y diferencia de funciones, derivada de un producto $\mathrm{y}$ de un cociente de funciones.

3. A nivel de objeto debe ser capaz de demostrar propiedades de la derivada de un mayor nivel de abstracción, es decir, funciones no representadas mediante una fórmula. Debe ser capaz de encapsular y desencapsular un proceso, es decir revertir un proceso, en el sentido de volver al proceso inicial, reflexionar sobre las acciones que al actuar sobre el proceso originaron el objeto. Además, coordinar nuevos procesos.

4. El estudiante debe ser capaz de coordinar el esquema función con el esquema de diferenciación y definir, desde este enfoque, los conceptos involucrados con el análisis cualitativo de funciones. Ésta coordinación les permitirá analizar y realizar dicho estudio vía diferenciación y revertir el proceso, es decir, conociendo el comportamiento de la función derivada inferir propiedades de la función. El estudiante reconoce y coordina ambos esquemas 
y lo aplica en la resolución de problemas de optimización. El estudiante reconoce, modela y resuelve problemas cuya representación matemática es una ecuación diferencial.

Etapa Intra: en este nivel el estudiante posee una colección de reglas para determinar derivadas y una colección de los conceptos que determinan las características cualitativas de una función y sus propiedades, conoce algoritmos de resolución de una ecuación diferencial lineal de primer orden, y reconoce cómo modelar los fenómenos que se representan mediante estas ecuaciones, pero no es capaz de reconocer las relaciones existentes entre ellos.

Etapa Inter: el estudiante comienza a reconocer que de alguna manera los esquemas se relacionan y que pueden mirarse como un todo. En este trabajo no se incluye la construcción del esquema de regla de la cadena, que ha sido descrito y explicado por Clark et al. (1997).

Etapa Trans: se caracteriza por la construcción de una estructura subyacente, coherente con algunas de las relaciones descubiertas en la etapa de desarrollo inter. El estudiante construye una estructura subyacente de conocimientos, propiedades de las funciones y reconoce varias instancias del concepto derivada, como derivadas de orden superior ligadas a un proceso. Además, construye la estructura formada por los distintos algoritmos de solución de una ecuación diferencial y los fenómenos que debe modelar. Los esquemas pasan a caracterizarse como una colección que se describe por un solo proceso.

\subsubsection{Módulos de Clases}

Todos los módulos tienen la misma estructura, exceptuando el primero que incluye un problema de entrada al capítulo, que representa el tipo de problemática que el estudiante debería ser capaz de resolver al final del curso. Al término de cada sección estos apuntes incluyen actividades ilustrativas y actividades grupales.

En el módulo $\mathrm{N}^{\circ} 1$, a nivel de acción se introduce el concepto de derivada evaluada en un punto, calculando una sucesión de variaciones medias en un intervalo cuya longitud tiende a cero, relacionándolas con la interpretación gráfica, de modo que en el límite se obtiene la derivada en un punto que coincide con la aproximación de la pendiente de la recta tangente en dicho punto. Además, se introduce y define el concepto de derivada numérica.

El concepto de proceso es un nivel más alto de comprensión que la acción, y se produce cuando el estudiante logra interiorizar las acciones, aplicando el concepto de derivada puede calcular la derivada de algunas funciones elementales, como funciones potenciales, polinómicas, inversas proporcionales, función raíz cuadrada. Además, puede realizar demostraciones simples usando la definición y las propiedades de los límites, determinar la 
derivada de la función exponencial y logarítmica, la derivada de una suma o de una diferencia. Estos conceptos y actividades son expuestos en el módulo $\mathrm{N}^{\circ} 2$.

El concepto de objeto es logrado a medida que el estudiante va reflexionando sobre las propiedades de la función derivada y realiza acciones sobre ella. Puede encapsular el proceso y desarrollar la comprensión de objeto derivada de una función. El concepto de encapsular un proceso es relevante en el sentido que en esta etapa el estudiante debería pasar fácilmente del objeto al proceso del cuál este proviene y viceversa, en tanto necesite trabajar con situaciones particulares. Debería ser capaz de extender el concepto y poder demostrar teoremas, usando las propiedades ya estudiadas, demostrar el teorema válido para la derivada de un producto y cociente para funciones en general, la derivada de la función inversa, y determinar derivadas de una composición de funciones elementales expresadas en forma más compleja. Además, de relacionar en un gráfico una función y sus primeras derivadas. Al construir mentalmente el objeto el estudiante puede extender el concepto al grupo de las funciones trigonométricas. Debería ser capaz de determinar la derivada de una función a partir de su gráfico, comprender los conceptos relacionados con el concepto de derivada como el concepto de diferencial de una función y sus aplicaciones al cálculo de aproximaciones. Estos conceptos fueron desarrollados en los módulos $\mathrm{N}^{\circ} 3, \mathrm{~N}^{\circ} 4$ y $\mathrm{N}^{\circ} 5$.

Con el constructo de objeto derivada, propiedades de una función y la demostración de propiedades, el estudiante comprende los conceptos y organiza en su mente un esquema individual para la derivada. El concepto de esquema involucra una colección de acciones, procesos, objetos y otros esquemas junto con sus relaciones, que el individuo comprende en relación a la derivada. En este momento, ha logrado desarrollar el nivel Intra de un esquema. Este esquema tendrá un significado si es capaz de relacionar estos conceptos y abordar con éxito cualquier fenómeno cuyo modelo matemático se vincule con el concepto de derivada, como la derivada de una función compuesta y sus aplicaciones a la derivación implícita y problemas de variables relacionadas. En este momento, ha alcanzado el nivel de pre-esquema del modelo cognitivo. Cuando haya construido una estructura subyacente de los conceptos y sus propiedades y vea el concepto ligado a ellos está logrando el nivel Trans. Debería ser capaz de resolver problemas de optimización y problemas de valor inicial (PVI), modelar fenómenos de crecimiento poblacional ilimitado y logístico, decaimiento radioactivo, segunda ley del enfriamiento de Newton, mezclas y disoluciones. Estos problemas que forman parte del currículo de los estudiantes son tratados en los módulos $\mathrm{N}^{\circ} 6$ y Nº7. 


\subsubsection{Laboratorios computacionales}

Se diseñaron cuatro laboratorios computacionales estructurados cognitivamente según APOS.

Laboratorio $N^{\circ} 1$. Objetivos: que el estudiante conozca los comandos e instrucciones básicas para realizar múltiples cálculos y operatoria básica; complementar y reforzar la teoría unida a la práctica usando gráficos, movimiento y color; visualizar, internalizar y transformar objetos desde un dominio abstracto a uno tecnológico más novedoso. Una acción es la reacción a un estímulo, por lo tanto, en este taller se estimulará el uso de la tecnología para realizar cálculos específicos, reforzar la comprensión de los conocimientos previos de los estudiantes desde la operatoria de la aritmética básica, expresiones algebraicas, resolución de ecuaciones hasta la definición de función y su representación gráfica.

Contenidos del Laboratorio $N^{\circ} 1$ : uso de los operadores y comandos básicos del álgebra; asignaciones; sumatorias y productos; ecuaciones; funciones (de bibliotecas y definidas por el usuario) y gráficos (de funciones, de figuras planas y puntos, gráficos animados).

Laboratorio $N^{\circ}$ 2. Objetivos: reforzar el concepto de la aproximación de la derivada en forma visual. La idea es que se perciba que este concepto no es estático, sino dinámico.

Contenidos del Laboratorio $N^{\circ} 2$ : Aplicaciones geométricas de la derivada en un punto; deslizamiento de la recta tangente a lo largo de una curva en los distintos puntos de la gráfica, a través del dominio de graficación seleccionado para representar la función, con el propósito de mostrar la idea de movimiento que no es posible de realizar en una pizarra; instrucciones que permiten resolver problemas relacionados con el cálculo de la derivada de una función e ilustración de problemas relacionados con el concepto y la metodología usada en el aula.

Laboratorio $N^{\circ} 3$. Objetivos: que el estudiante aplique los conocimientos adquiridos en los laboratorios anteriores, de modo que pueda ir en forma natural del concepto objeto al de proceso y viceversa. Este taller se diseñó a un nivel más alto de comprensión. Debería resolver problemas aplicados usando el concepto, propiedades de la derivada, realizar el estudio analítico de una función y resolver problemas de tasa de cambio.

Contenidos del Laboratorio $N^{\circ} 3$ : comandos e instrucciones que se requieren para resolver un problema de razón de cambio; resolución de un problema modelo resuelto paso a paso, según la metodología usada en el aula usando las instrucciones que proporciona el software, problemas propuestos para que los estudiantes realicen en forma grupal.

Laboratorio $N^{\circ}$ 4. Objetivos: está orientado a integrar el software con problemas de complejidad superior. Si el estudiante ha adquirido la concepción de objeto derivada y 
conceptos afines relacionados con las propiedades de la derivada y estrategias de cálculo, debería ser capaz de organizar la información, revertir procesos y resolver problemas, extender la potencialidad del software, que incluye desde la situación gráfica del problema, la resolución del modelo matemático y la respuesta al problema contextualizado. Se trata de incentivar la creatividad del estudiante mediante situaciones que involucren gráficos en tres dimensiones.

Contenidos del Laboratorio $N^{\circ} 4$ : Aplicar el software a la resolución de problemas de optimización y problemas de tasa de cambio de una función real de una variable real.

\subsubsection{Instrumentos de obtención de información}

Se usaron dos instrumentos. Uno es el conjunto de evaluaciones aplicadas durante el curso de la asignatura de matemáticas I, denominado instrumento secundario, usado solo como referencia. El otro es la Prueba de Medición de Estándares (PME) que fue el instrumento primario y que no forma parte del conjunto de evaluaciones de la asignatura de matemáticas I. La PME se diseñó aplicando el modelo cognitivo APOS que permitió analizar y discutir el aprendizaje construido de los casos seleccionados en esta investigación. Los temas considerados para construir la PME se seleccionaron del conjunto de estándares de aprendizajes para un curso de cálculo diferencial e integral ${ }^{8}$. (Ver anexo1)

\subsubsection{Instrumento de análisis}

La descomposición genética de la PME considera a nivel de acción reconocer el concepto de derivada en un punto (P1), la interpretación geométrica y el concepto de función derivada (P2) y (P3) respectivamente, a nivel de proceso. El cálculo de la derivada de una función elemental, indicador (P4), a un nivel de objeto. A nivel de esquema-Inter, la aplicación de la derivada al análisis cualitativo de funciones (P5), y la interpretación y lectura de un gráfico relacionado con una interpretación física de la derivada (P6). También, en este nivel, reconocer los indicadores (P7) y (P8) que corresponden a una aplicación de la primera derivada a los valores extremos y puntos de inflexión de una función y el indicador (P9) que relaciona la segunda derivada con los valores extremos y puntos de inflexión de una función.

\footnotetext{
${ }^{8}$ Estos estándares son el resultado del proyecto MECESUP UCH0002 (ALTIMIRAS et al., 2004) mediante el cual se fijaron estándares terminales de aprendizaje con el fin de homogeneizar los contenidos de las mallas curriculares en matemáticas de los estudiantes que pertenecen a 5 facultades de la Universidad de Chile.
} 
En este nivel el estudiante requiere de una mayor comprensión para relacionar los distintos esquemas involucrados. El identificar un modelo y la resolución de problemas que se representan mediante una ecuación diferencial (P10), es un nivel de comprensión en el que debe coordinar varios esquemas, se define, entonces, a nivel de esquema al menos Inter, y cuando el estudiante adquiere con claridad la estructura que se subyace al concepto de derivada transita al nivel Trans. El instrumento de análisis se presenta en el Cuadro 1, que considera los estándares seleccionados, los indicadores de logro asociados a estos estándares, las alternativas correctas de la prueba de estándares y su relación con la descomposición genética.

\begin{tabular}{|l|c|c|l|}
\hline Estándares de Aprendizaje & $\begin{array}{l}\text { Indicadores de } \\
\text { logro }\end{array}$ & $\begin{array}{l}\text { Alternativas } \\
\text { correctas }\end{array}$ & $\begin{array}{l}\text { Comprensión } \\
\text { según APOS }\end{array}$ \\
\hline $\begin{array}{l}\text { Comprende el concepto de derivada y su } \\
\text { interpretación }\end{array}$ & P1 & v) & Acción \\
\hline $\begin{array}{l}\text { Comprende el concepto de derivada y su } \\
\text { interpretación }\end{array}$ & P2 & Proceso \\
\hline Conoce la definición formal de derivada & P3 & iv) & Proceso \\
\hline Usa correctamente las reglas de derivación & P4 & iv) & Objeto \\
\hline Analiza funciones mediante el cálculo diferencial & P5 & Esquema \\
\hline Analiza funciones mediante el cálculo diferencial & P6 & abierta & Esquema \\
\hline Comprende y aplica criterios de optimalidad & P7 & i) & Esquema \\
\hline Comprende y aplica criterios de optimalidad & P8 & iii) & Esquema \\
\hline Comprende y aplica criterios de optimalidad & P9 & i) & Esquema \\
\hline $\begin{array}{l}\text { Es capaz de modelar y resolver situaciones concretas } \\
\text { mediante ecuaciones diferenciales }\end{array}$ & $\begin{array}{l}\text { P10 } \\
\text { iii) }\end{array}$ & Esquema \\
\hline
\end{tabular}

Cuadro 1 - Instrumento de Análisis. $\mathrm{P}_{\mathrm{i}}$ denota el problema número i

Fuente: desarrollado por los autores

Los resultados obtenidos en la PME se presentan codificados en la Sección 4, donde 1 es logrado y 0 es no logrado. El objetivo principal de la PME fue conocer el nivel de comprensión alcanzado por los estudiantes en el aprendizaje del concepto de derivada y algunas de sus aplicaciones, usando el modelo cognitivo propuesto por Dubinsky. Esta prueba se aplicó ocho meses después de la dictación del curso.

\section{Análisis y Discusión de los Resultados}

\subsection{Evaluaciones y resultados de rendimiento}

En la Tabla 1 se presentan como referencia las calificaciones que obtuvieron las estudiantes en la asignatura Matemáticas I. La calificación semestral está formada por un conjunto de 4 tipos de evaluaciones: parciales que reflejan un dominio parcial de los 
contenidos (10\%), dos pruebas acumulativas que consideran una mayor cantidad de contenidos (70\%) y el promedio de los laboratorios (20\%).

Tabla 1 - Calificaciones Asignatura Matemáticas I

\begin{tabular}{lccc}
\hline & Calificación Semestral & Examen & Calificación Final \\
\hline Daniela & 3.8 & 4.2 & 4.0 \\
Lola & 5.0 & & 5.0 \\
\hline
\end{tabular}

Fuente: desarrollado por los autores

El reglamento de calificaciones, contempla que un estudiante con calificación semestral mayor o igual que 5.0 queda eximido de rendir el examen. Observamos, de la Tabla 2, que ambas estudiantes aprobaron la asignatura. Daniela obtuvo una calificación final suficiente y Lola aprueba con calificación 5.0, eximiéndose del examen. Estos resultados fueron una referencia en este estudio, puesto que no se consideró el nivel de aprendizaje construido en el transcurso de la asignatura.

\subsection{Análisis}

La Tabla 2 muestra los logros obtenidos mediante la PME.

Tabla 2 - Resultados de la PME Codificados

\begin{tabular}{lcccccccccc}
\hline Estudiantes & P1 & P2 & P3 & P4 & P5 & P6 & P7 & P8 & P9 & P10 \\
Daniela & 0 & 1 & 1 & 1 & 0 & 0 & 1 & 1 & 1 & 0 \\
Lola & 0 & 0 & 1 & 1 & 1 & 0 & 1 & 1 & 1 & 1 \\
\hline \multicolumn{8}{c}{ Fuente: desarrollado por los autores }
\end{tabular}

Se indagará de sus respuestas el nivel de aprendizaje construido según el modelo APOS.

Caso 1. Daniela: corresponde al perfil de una estudiante tímida, insegura y temerosa de usar la tecnología, puesto que nunca había trabajado en un ordenador en forma autónoma. Su rendimiento en la asignatura de Matemáticas I fue suficiente, le cuesta aprender matemáticas. Se observa de la Tabla 3, que el número de respuestas correctas es 6 de un total de 10 . Analizamos los argumentos de una de respuesta incorrecta y una correcta.

Pregunta 1. Es un indicador de reconocimiento y comprensión del concepto de derivada. Daniela marca en la hoja de repuesta la alternativa correcta, sin embargo su explicación es incorrecta y su argumento es contradictorio.

Ella es uno de los casos más comunes de los estudiantes que responden bien y justifican mal. Esto evidencia que conoce el concepto a nivel de acción, lo memorizó sin comprenderlo, falla porque relaciona el concepto de derivada con la pendiente de una recta secante. 
La respuesta a este indicador se consideró incorrecta y el argumento de Daniela es el siguiente:

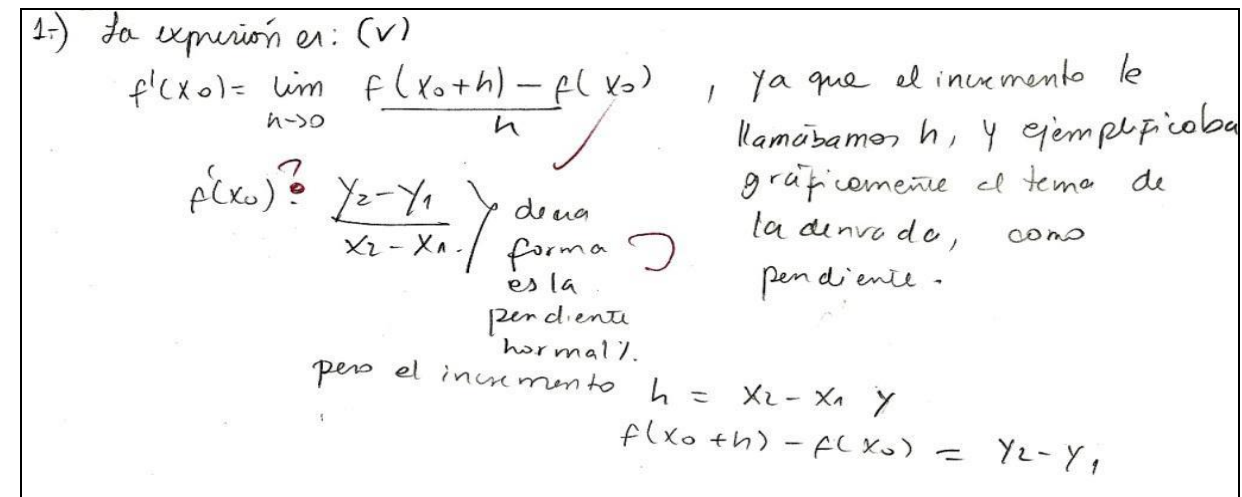

Figura 1 - Respuesta de Daniela al indicador $\mathrm{N}^{\mathrm{o}} 1$ Fuente: datos de la investigación

Pregunta 4. Daniela aplica las propiedades para determinar la derivada de una función potencial, evidenciando su conocimiento y manejo algebraico. Este es un indicador simple, que se clasificó a nivel de objeto derivada y que respondió en forma correcta.

$\begin{aligned} \text { 4) } y=\frac{1}{12} x^{6} & \frac{d y}{d x}=\frac{6}{12} x^{5}=\frac{1}{2} x^{5} \\ \text { alternation } & \text { IV) } y^{\prime}=\frac{1}{2} x^{5}\end{aligned}$

Figura 2 - Respuesta de Daniela al indicador $\mathrm{N}^{\circ} 4$

Fuente: datos de la investigación

\section{Informe de la PME}

Del resultado total evidenciado en la PME se observa que Daniela justifica en forma correcta 6 respuestas de 10. Daniela evidencia conocimiento de los indicadores $\mathrm{N}^{\circ} 2, \mathrm{~N}^{\circ} 3$ (acción) y $\mathrm{N}^{\circ} 4$ (objeto). Además, se deduce de sus respuestas a los indicadores $\mathrm{N}^{\circ} 7, \mathrm{~N}^{\circ} 8$ y $\mathrm{N}^{\circ} 9$ que es capaz de relacionar los esquemas de derivada, propiedades cualitativas de una función y graficación, aunque algunas de sus justificaciones son escuetas. De acuerdo al modelo cognitivo, ha desarrollado un aprendizaje a nivel de pre-esquema.

Caso 2. Lola: su perfil corresponde a una estudiante con habilidades matemáticas, comprende fácilmente los nuevos conceptos, lo que se deduce de su participación en las discusiones en el aula con sus compañeros, interviniendo cuando se plantean interrogantes. Tiene destreza para trabajar con el software y el ordenador, y le gusta sociabilizar sus conocimientos. De la Tabla 3 se observa que Lola responde 7 indicadores de un total de 10.

Pregunta 2. Su respuesta en relación al indicador $\mathrm{N}^{\circ} 2$ es incorrecta. Este indicador mide el reconocimiento y la comprensión de la derivada en un punto y su interpretación geométrica. 
Lola confunde en el gráfico la representación de la pendiente de la recta tangente en un punto con la pendiente de la recta secante que pasa por dos puntos. Su justificación es:

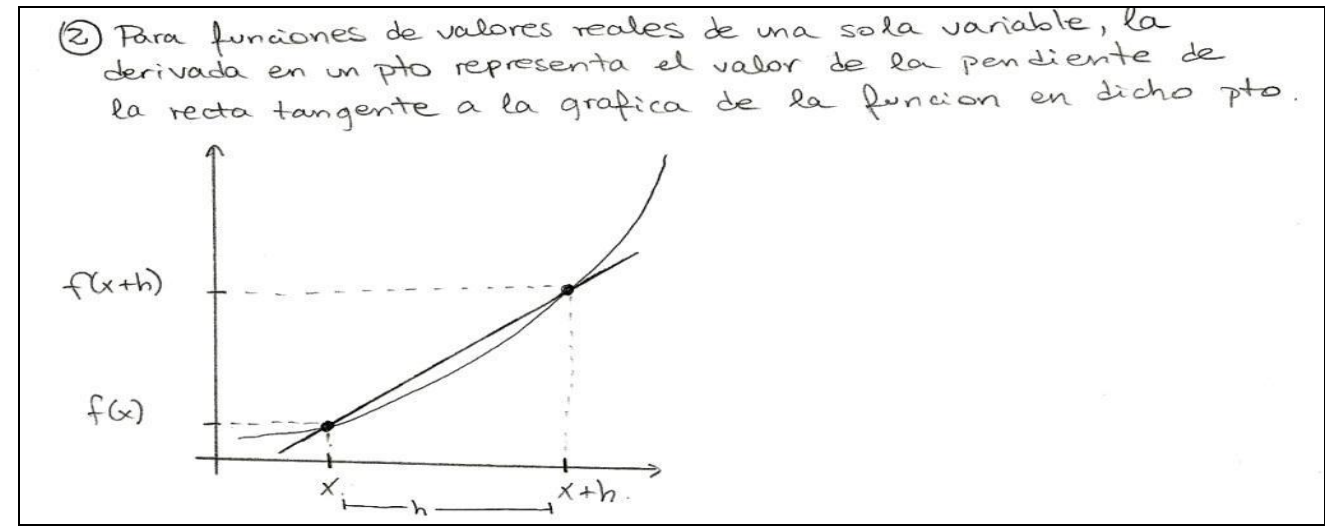

Figura 3 - Respuesta de Lola al indicador $\mathrm{N}^{\circ} 2$ Fuente: datos de la investigación

Pregunta 5. Integra y relaciona varios conceptos relacionados con la derivada. Se clasificó a nivel de esquema-Inter y Lola responde en forma correcta. Lola relaciona geométricamente los conceptos: función creciente, signos de la primera y segunda derivada, y la forma gráfica. Muestra evidencia de integrar correctamente estos conceptos. Su justificación es:

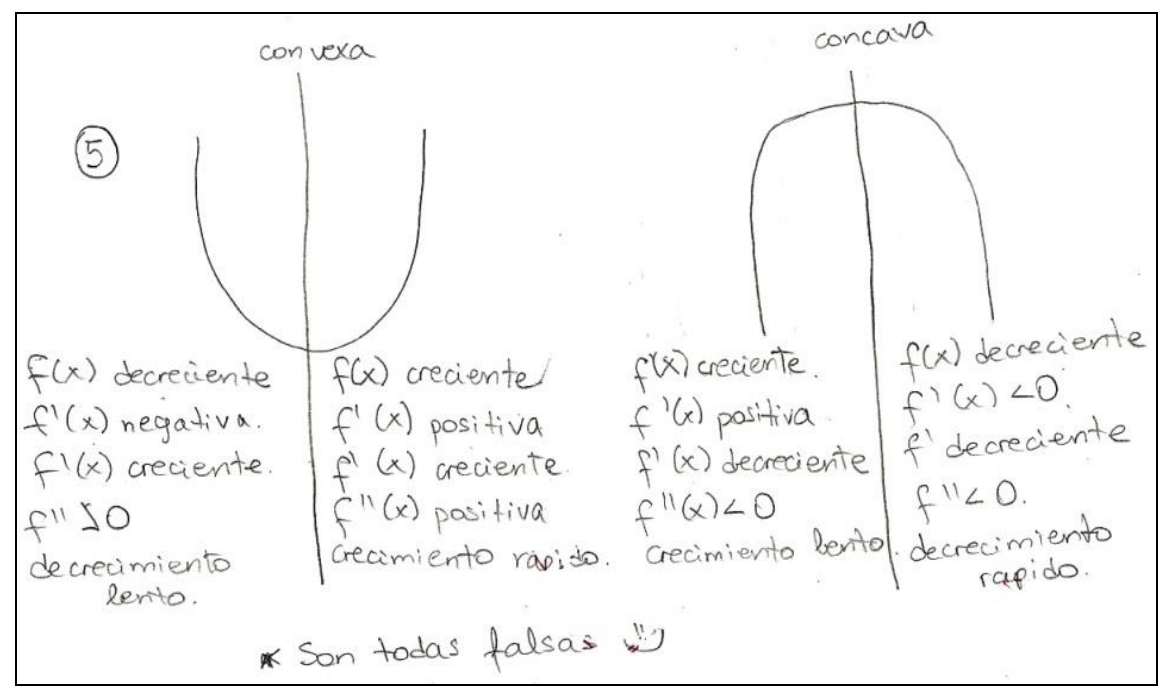

Figura 4 - Respuesta de Lola al indicador No5 Fuente: datos de la investigación

\section{Informe de la PME}

Lola revela un aprendizaje débil del concepto de derivada en un punto (nivel de acción). Sin embargo, evidencia un aprendizaje a nivel de pre-esquema del concepto de derivada, en relación al análisis cualitativo de funciones y a la resolución de problemas. 


\subsection{Relación entre los resultados de la asignatura y la PME}

Al comparar los logros obtenidos en la PME con los resultados de la asignatura se puede deducir que:

Caso 1. Daniela: ha desarrollado un aprendizaje a nivel de pre-esquema débil, logró relacionar solo dos esquemas, el de la derivada con el análisis cualitativo de funciones, si las preguntas se formulan en forma directa. No logró integrar el esquema de problemas que se modelan mediante la ecuación logística. Daniela aprobó la asignatura de Matemáticas I con la nota mínima de aprobación. Se podría conjeturar que aprobar una asignatura de matemáticas con calificación suficiente, significa que el estudiante logra coordinar solo algunos esquemas relacionados con un determinado tópico matemático. Se observa que existe concordancia entre esta calificación y los conocimientos evidenciados en la PME. Es destacable que los conocimientos que ella adquirió perduraran en el tiempo.

Caso 2. Lola: su calificación final en la asignatura de Matemáticas I fue 5.0. Hay una correlación directa entre esta calificación y el aprendizaje evidenciado en la PME. Se puede inferir que el aprendizaje construido por Lola corresponde, en el modelo cognitivo, a un preesquema, con debilidad en la comprensión de conceptos teóricos básicos provenientes de las etapas de acción y proceso. Se puede conjeturar, en este caso, que esta debilidad le ha impedido transitar a una etapa de aprendizaje a nivel Trans del concepto mirado globalmente, es decir, como afirma Dubinsky (1991) ver el concepto y sus aplicaciones como un todo.

\section{Conclusiones}

\subsection{Síntesis de los resultados}

El análisis muestra la evidencia del aprendizaje construido, evaluado después de 8 meses del término del curso. El modelo APOS ha permitido evidenciar las etapas logradas por cada estudiante. Los módulos de clases y laboratorios computacionales cumplieron distintas funciones, según las habilidades personales, subsunsores y experiencia previa de las alumnas. Caso 1. Daniela: respondió correctamente los indicadores $\mathrm{N}^{\circ} 2$ al $\mathrm{N}^{\circ} 4, \mathrm{y} \mathrm{N}^{\circ} 7, \mathrm{~N}^{\circ} 8$ y $\mathrm{N}^{\circ} 9$. Logró un desarrollo débil de objeto derivada, puede manipular la operatoria simple. Sin embargo, sus argumentos para justificar las respuestas a los indicadores $\mathrm{N}^{\circ} 2$ y $\mathrm{N}^{\circ} 3$ no son directos. No obstante, es capaz de relacionar esquemas. En conclusión, el aprendizaje construido por Daniela a nivel de pre-esquema es débil, en el sentido que maneja algunos 
conceptos a nivel de objeto y muestra debilidad en algunos procesos inversos, por tal motivo no logró un desarrollo completo de pre-esquema. Desde el enfoque de la abstracción reflexiva, la estudiante ha acomodado su esquema mental existente para manejar el concepto y aplicarlo a algunas propiedades en forma aislada. Se aprecia una correlación entre las calificaciones de referencia y la PME.

Caso 2. Lola: respondió correctamente los indicadores: $\mathrm{N}^{\circ} 3, \mathrm{~N}^{\circ} 4$ y $\mathrm{N}^{\circ} 5$, y desde el $\mathrm{N}^{\circ} 7$ al $\mathrm{N}^{\circ} 10$. En sus argumentos confunde el concepto de derivada en un punto con el concepto de función derivada. No comprendió significativamente ambas definiciones, su comprensión fue débil, por lo tanto, no perduró en el tiempo. La etapa de reflexión evita la confusión entre conceptos. El repetir y reflexionar una acción permite desarrollar el proceso derivada y en esta etapa Lola demuestra ambigüedad. Sin embargo, logra manejar la operatoria asociada a la función derivada y sus relaciones con las propiedades cualitativas de una función, lo que permite deducir que ha desarrollado en estos temas un aprendizaje a nivel de pre-esquema. Maneja con destreza la operatoria y es capaz de relacionar esquemas. Adicionalmente, relaciona los esquemas involucrados en la resolución de problemas que se modelan mediante la ecuación logística. Desde la abstracción reflexiva, Lola ha logrado asimilar las distintas aplicaciones del concepto, a pesar del conflicto epistemológico detectado.

\subsection{Conclusiones sobre el aprendizaje evidenciado por APOS}

Uno de los objetivos al realizar esta investigación era conocer qué procesos cognitivos realiza un sujeto para aprender matemáticas. Específicamente, indagar el nivel de aprendizaje construido en el estudio de la derivada y algunas de sus aplicaciones, lo que nos permitió percibir cuáles son las dificultades que enfrentan algunos estudiantes en su aprendizaje.

Se logró determinar que si a nivel de acción, el concepto de la derivada en un punto no es comprendido y el estudiante no reflexiona sobre él, tiene dificultades para extenderlo y transitar al nivel de proceso, puesto que no es capaz de relacionar correctamente su significado y realizar la abstracción que relaciona este concepto con la definición de la función derivada. Además, se verificó la dificultad que muestra el estudiante para interpretar geométricamente el concepto. La conclusión a este respecto es que las estudiantes lograron el nivel de proceso en su oportunidad, de lo contrario no podrían haber avanzado al nivel de objeto y posteriormente al de esquema. Además, lo que ha perdurado en su mente a través del tiempo son las propiedades de la operatoria y la interpretación gráfica del concepto, es decir, el concepto operacional y visual por sobre el concepto formal. Esta conclusión se fundamenta 
en el hecho de que los casos seleccionados respondieron correctamente los indicadores $\mathrm{N}^{\circ} 3 \mathrm{y}$ $\mathrm{N}^{\circ} 4$, que se definieron a nivel de proceso y objeto respectivamente al aplicar el modelo APOS. El indicador $\mathrm{N}^{\circ} 3$ muestra que el estudiante es capaz de reconocer la definición formal del concepto de función derivada. El conocimiento de este concepto, implica tener otros conocimientos previos y habilidades relacionadas con él, como saber calcular límites al infinito, y comprender que, calculando un límite al infinito, se obtiene una función denominada función derivada y un número denominado derivada en un punto. $\left(\mathrm{f}^{\prime}\left(x_{0}\right)=\lim \frac{f\left(x_{0}+h\right)-f\left(x_{0}\right)}{h}\right)$. Concluimos que ambas estudiantes lograron estas competencias.

El indicador $\mathrm{N}^{\circ} 4$ mide habilidad en la operatoria (objeto). El estudiante debe ser capaz de ir del concepto a la aplicación y viceversa. Se verificó que las estudiantes son capaces de aplicar y manejar el concepto sin influencias externas. Se concluye que, a pesar de que ambas no reconocieron el concepto de derivada en un punto (P1), esto no fue un obstáculo para que respondieran correctamente (P4), lo que implica conocer y aplicar propiedades del concepto.

El indicador $\mathrm{N}^{\circ} 5$ ha evidenciado que algunos estudiantes tienen dificultades de comprensión en aquellos enunciados en que deben discriminar entre proposiciones falsas y verdaderas, puesto que los indicadores siguientes (P7, P8 y P9) demuestran lo contrario. Integran el esquema de la derivada con el de las propiedades cualitativas de las funciones. Las dos estudiantes respondieron justificando correctamente sus respuestas. El indicador $\mathrm{N}^{\circ} 6$ plantea la relación entre posición y rapidez de cambio en forma gráfica, es una aplicación simple de la Física, que respondió una de las estudiantes. El indicador $\mathrm{N}^{\circ} 9$ relaciona la segunda derivada con valores extremos y punto de inflexión, ambas coordinaron correctamente los esquemas. El indicador $\mathrm{N}^{\circ} 10$ mide si el estudiante es capaz de reconocer y resolver la ecuación logística. Este tipo de problemática integra esquemas del cálculo diferencial y del cálculo integral. Se concluye que una de ellas logró en esta aplicación un aprendizaje de nivel esquema-Trans.

\subsection{De la metodología de la investigación y APOS}

1. Una conclusión global que se deduce al usar este modelo es que, a pesar de las dificultades de comprensión de algunos conceptos básicos, teóricos, definidos a nivel de acción, las estudiantes lograron consolidar aprendizajes de nivel superior y fueron capaces de 
relacionar conceptos e integrar el concepto derivada con otros esquemas. Esta conclusión coincide con la de Artigue (1995) mencionada también por Sánchez, Garcia y Llinares (2008).

2. Las conclusiones referentes al nivel de aprendizaje construido por cada estudiante evidencian que coordinan al menos uno de los esquemas involucrados, alcanzando un aprendizaje a nivel de pre-esquema, cada una en distinto grado. Se observa que los resultados son coincidentes con los de Sánchez, Garcia y Llinares (2008, p.287) en el sentido que: "el desarrollo de esquema de derivada no es algo vinculado a conocer muchos elementos constitutivos del concepto, sino ser capaces de coordinarlos al resolver problemas".

3. Se percibe, al igual que en el estudio de Parraguez (2009), la característica de no linealidad del aprendizaje en cada caso. Se observó que la concepción de derivada puede mostrar una idea específica para explicar monotonía y si se agregan otros elementos como, por ejemplo, la concavidad que está relacionada específicamente con la forma de la gráfica de una función, se percibe que estos conceptos son relacionados e integrados como parte de la concepción de derivada.

4. Se observa una correlación entre los resultados obtenidos mediante el instrumento secundario y la Prueba de medición de Estándares. Esta correlación se percibe al comparar la similitud de las calificaciones obtenidas por ambos instrumentos.

\subsection{De la estrategia de enseñanza}

1. Los recursos didácticos implementados como parte de la metodología de enseñanza juegan un rol importante en el desarrollo de la comprensión, permiten que el estudiante relacione más de un esquema. La síntesis de la información gráfica y analítica se produce al menos a nivel de pre-esquema. Sánchez, García y Llinares (2008) sostienen que se produce a nivel de esquema-Trans.

2. Los aprendizajes adquiridos durante el curso de Matemáticas I han perdurado en el tiempo significativamente en cada estudiante. Conjeturamos que el recurso didáctico ha sido un gran apoyo en el aprendizaje. Resultados similares aporta Trigueros (2005).

3. Respecto del entorno computacional como una herramienta de apoyo al aprendizaje se conjetura que es una de las variables que ha incidido en obtener un aprendizaje que ha perdurado en el tiempo, idea compartida con los autores Codes y Sierra (2005). 


\section{Agradecimientos}

Subvencionado por el Centro de Investigación Avanzada en Educación (CIAE) (Proyecto PBCT CIE-05, 2012-2013).

\section{Referencias}

ALTIMIRAS, L.; DARTNELL, P.; FELMER, P.; GALAZ, M.; HUMERES, M. E.; VEGA, M. A.; YAÑEZ, M. F. Estándares para un curso de Cálculo Diferencial e Integral. Santiago de Chile: Naval Editora e Imprenta, 2004.

ANESHKUMAR, M. An APOS analysis of students' understanding of the concept of a limit of a function. Phythagoras, Durvanville SA, v. 71, p. 41-52, July 2010.

ARTIGUE, M. La enseñanza de los principios del cálculo: problemas epistemológicos, cognitivos y didácticos. En: GÓMEZ, P. (Ed.). Ingeniería didáctica en educación matemática: Un esquema para la investigación y la innovación en la enseñanza y el aprendizaje de las matemáticas. Bogotá: Grupo Editorial Iberoamericano, 1995. p. 97-140.

ASIALA, M.; BROWN, A.; DE VRIES, D.; DUBINSKY, E.; MATHEWS, D.; THOMAS, K. A framework for research and curriculum development in undergraduate mathematics education. CBMS Issues in Mathematics Education, Providence R.I, v. 6, p. 1-32, 1996.

AUSUBEL, D.; NOVAK, J. D.; HANESIAN, H. Psicología Educativa: Un punto de vista cognoscitivo. Ciudad de México: Ed. Trillas, 1978.

BARBOSA, K. La enseñanza de las inecuaciones desde el punto de vista de la teoría APOS. Revista Latinoamericana de Investigación en Matemática Educativa, Ciudad de México, v. 6, n. 3, p. 199219, 2003.

BETH, E. W.; PIAGET, J. Epistemología Matemática y Psicología: relaciones entre la lógica formal y el pensamiento real. Barcelona: Editorial Crítica Grijalbo, 1980.

CLARK et al. Constructing a schema: The case of the chain rule. Journal of Mathematical Behavior, Amsterdam, v. 16, n. 4, p. 345-364, 1997.

CODES, M.; SIERRA, M. Entorno computacional y educación matemática: Una revisión del estado actual. SIMPOSIO SEIEM, 9., 2005, Córdoba. Anales... Córdoba; Didáctica del análisis. 2005.

DUBINSKY, E. Reflective abstraction in advanced mathematical thinking. In: TALL, D. Advanced Mathematical thinking. Dordrecht: Kluwer, 1991. p. 95-123.

DUBINSKY, E. El aprendizaje de los conceptos abstractos de la matemática avanzada: Memorias de la X REUNIÓN CENTROAMERICANA Y DEL CARIBE sobre formación de profesores e investigación en matemática educativa. San Juan Bautista de Puerto Rico: Universidad de Puerto Rico, 1996. p. 1-9.

PARRAGUEZ, M. Evolución cognitiva del concepto de espacio vectorial. 2008. 250 f. Tesis (Doctorado en Matemática Educativa) - Instituto Politécnico Nacional, Centro de Investigación en Ciencia Aplicada y Tecnología Avanzada, Ciudad de México, 2009. 
PIAGET, J. The Equilibration of cognitive structures. Cambridge: Harvard University Press, 1985. (Original published 1975)

PIAGET, J.; GARCÍA, R. Psychogenesis and the history of science. New York: Columbia University Press, 1983.

SÁNCHEZ, G.; GARCÍA, M.; LLINARES, S. La comprensión de la derivada como objeto de investigación en didáctica de la matemática. Revista Latinoamericana de Investigación en Matemática Educativa, Ciudad de México, v. 11, n. 2, p. 267-296, 2008.

SFARD, A. Reification as a birth of a metaphor. For the learning of mathematics. Fredericton N. B., v. 14 , n. 1 , p. $44-55,1994$.

TALL, D.; VINNER, S. Concept images and concept definition in mathematics with special references to limit and continuity. Educational Studies in Mathematics, Berlin, n. 12, p. 151-169, 1981

TRIGUEROS, M. La noción de esquema en la investigación matemática educativa a nivel superior. Educación Matemática, Ciudad de México, v. 17, n. 1, p. 5-36, 2005.

VEGA, M. A. El Análisis de la construcción del concepto de la derivada en un primer ciclo de enseñanza superior asistida por ordenador. 2011. 439 f. Tesis (Doctorado en Educación) - Facultad de Ciencias de la Educación, Universidad de Huelva, Huelva, 2012.

Submetido em Maio de 2013. Aprovado em Junho de 2013. 


\section{ANEXO 1: Estándares de Aprendizaje y Prueba de Medición de Estándares (PME)}

Los estándares de aprendizaje requieren que se explicite lo que se espera que los estudiantes sepan y sean capaces de hacer. Nacen de la necesidad de responder a la pregunta de cuán adecuado es el desempeño del estudiante. Los estándares van acompañados de indicadores que permiten medir si los objetivos han sido alcanzados. Los estándares además de homogeneizar los contenidos programáticos de las mallas curriculares en matemáticas permiten que a priori que los estudiantes conozcan lo que se espera de ellos y puedan saber en todo momento su nivel de avance y a qué distancia están de lograrlos propósitos del curso ${ }^{9}$.

\subsection{Estándares para la sección de Cálculo Diferencial}

Como objetivo terminal se espera que a través de los indicadores de logro el alumno:

1. Comprenda el concepto de derivada y sus interpretaciones. (ALTIMIRAS et al., 2004) (p. 55)

2. Conozca y maneje la definición formal de derivada. (Ibid. p. 56)

3. Aproxime raíces de ecuaciones usando el método de Newton. (Ibid. p.57)

4. Comprenda el teorema: Toda función diferenciable es continua. (Ibid. p. 58)

5. Use correctamente las reglas de derivación. (Ibid. p. 59)

6. Comprenda el teorema del valor medio para las funciones diferenciables. (Ibid, p. 61)

7. Analice límites usando la regla de L'Hopital. (Ibid. p. 62)

8. Analice funciones mediante el cálculo diferencial ${ }^{10}$. (Ibid. p. 63)

9. Comprenda y aplique criterios de optimalidad. (Ibid. p. 64)

10. Sea capaz de modelar problemas con variables relacionadas. (ibid. p. 65)

11. Sea capaz de modelar resolver situaciones concretas mediante ecuaciones diferenciales. (Ibid.p.66)

\subsection{Prueba de medición de Estándares}

Se seleccionaron los estándares relacionados con el concepto formal de la derivada,

\footnotetext{
${ }^{9}$ Un estándar es una declaración de aprendizaje esperado y uno o más ejemplos de problemas que permitan decidir si éste se ha logrado. PROYECTO MECESUP UCH0002

${ }^{10}$ Se refiere al análisis cualitativo de funciones usando Cálculo Diferencial.
} 
cálculo de la derivada de funciones elementales, aplicación de la derivada al análisis cualitativo de una función y el modelado y resolución de problemas que se representan mediante una ecuación diferencial. Es decir, aquellos relacionados con el reconocimiento y comprensión del concepto en estudio, operación básica y dos aplicaciones importantes que servirán de base para el curso siguiente, la asignatura Matemáticas II.

Se presenta a continuación la Prueba de Medición de Estándares que se aplicó.

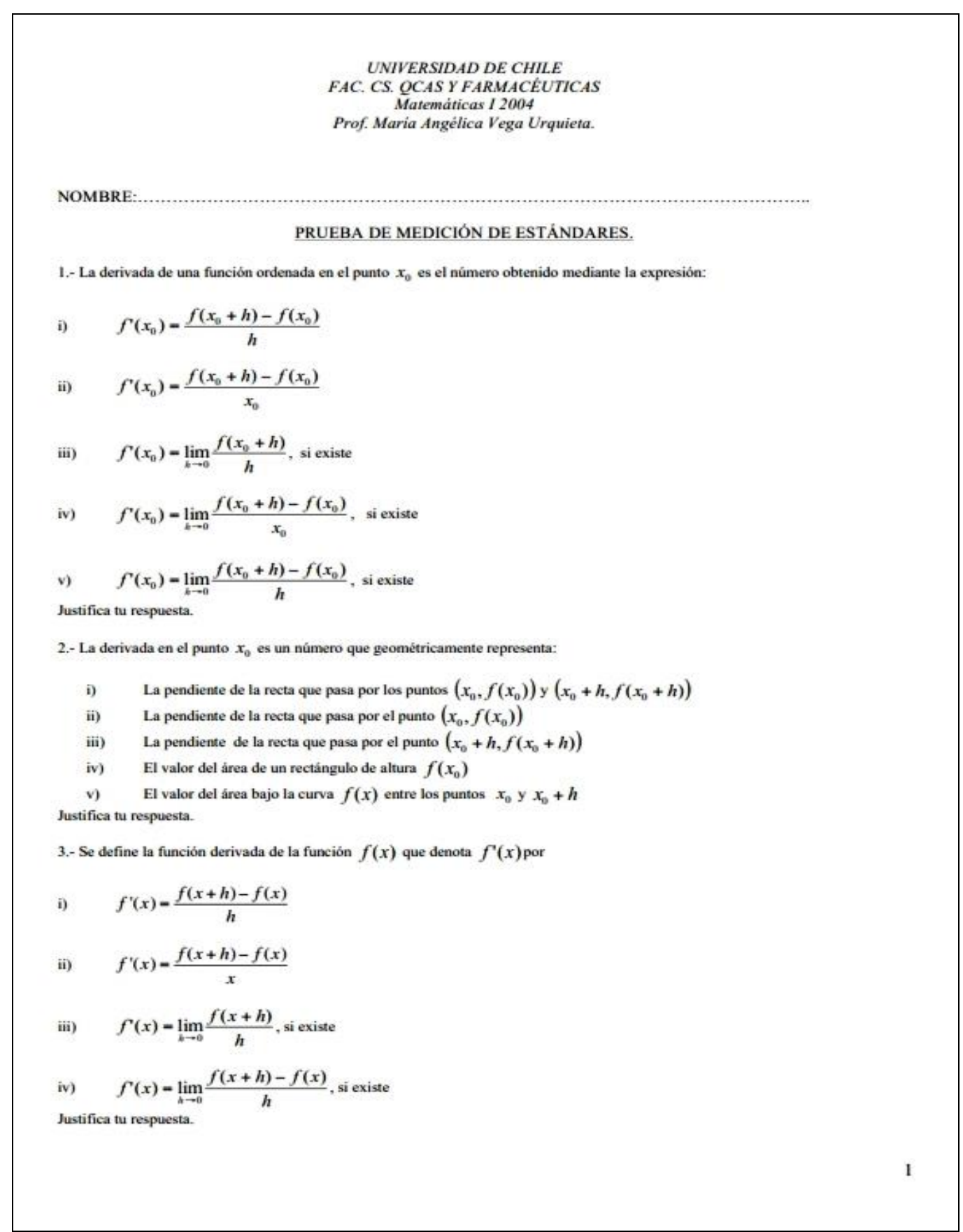

Figura 5 - PME - Indicadores 1 a 3

Fuente: desarrollado por los autores 
4.- $y=\frac{1}{12} x^{6}$, entonces la función derivada es:

i) $y^{\prime}=6 x^{5}$

ii) $y^{\prime}=\frac{1}{12} \frac{x^{7}}{7}=\frac{1}{84} x^{7}$

ii) $y^{\prime}=\frac{x^{6}}{72}$

iv) $y^{\prime}=\frac{1}{2} x^{5}$

v) $y^{\prime}=\frac{1}{72} x^{5}$

Justifica tu respuesta.

5.- ¿Cuál (Cuáles) de las siguiente(s) informacione(s) es (son) verdadera(s)?,

i) Si $f(x)$ es creciente en $[a, b], f^{\prime}(x)>0$ y la grafica es cóncava entonces $f^{\prime}(x)$ es creciente.

ii) Si $f(x)$ es creciente en $[a, b], f(x)>0$ y la gráfica convexa entonces $f(x)$ es decreciente.

iii) Si $f(x)$ es decreciente en $[a, b], f^{\prime}(x)<0$ y la gráfica es cóncava $\Rightarrow f^{\prime}(x)$ es creciente.

iv) Si $f(x)$ es decreciente en $[a, b], f^{\prime}(x)<0$ y en la grafica convexa entonces $f^{\prime}(x)$ es decreciente.

v) Ninguna de las anteriores.

Justifica tu respuesta.

6.- El siguiente es el gráfico de la posición de un móvil que se mueve en línea recta, ¿Qué sucede con el móvil cuando el grafico alcanza la cresta de una loma.

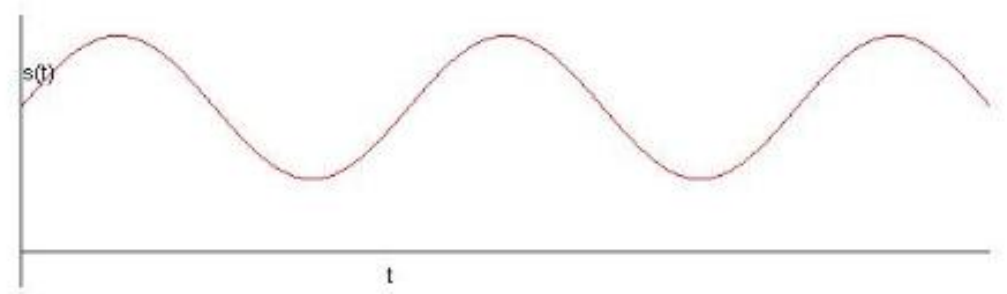

Justifica tu respuesta.

7.- Si la derivada de una función $f(x)$ al cruzar el punto $x_{0} \in D f$ cambia de positiva a negativa implica que:

$\begin{array}{ll}\text { i) } & \left(x_{0}, f\left(x_{0}\right)\right) \text { es un máximo relativo } \\ \text { ii) } & \left(x_{0}, f\left(x_{0}\right)\right) \text { es un máximo absoluto } \\ \text { iii) } & \left(x_{0}, f\left(x_{0}\right)\right) \text { es un mínimo relativo } \\ \text { iv) } & \left(x_{0}, f\left(x_{0}\right)\right) \text { es un minimo absoluto } \\ \text { v) } & \left(x_{0}, f\left(x_{0}\right)\right) \text { es un punto de inflexión }\end{array}$

Justifica tu respuesta.

Figura 6 - PME - Indicadores 4 a 7

Fuente: desarrollado por los autores 
8.- Si la derivada de una función $f(x)$ al cruzar el punto $x_{0} \in D f$ cambia de negativa a positiva implica que-

i) $\quad\left(x_{0}, f\left(x_{\mathrm{p}}\right)\right)$ es un máximo relativo

ii) $\quad\left(x_{0}, f\left(x_{0}\right)\right)$ es un máximo absoluto

iii) $\quad\left(x_{\mathrm{v}}, f\left(x_{\mathrm{o}}\right)\right)$ es un minimo relativo

iv) $\quad\left(x_{0}, f\left(x_{0}\right)\right)$ es un minimo absoluto

v) $\quad\left(x_{0}, f\left(x_{0}\right)\right)$ es un punto de inflexión.

Justifica tu respuesta

9.- ¿Cuál de las siguientes afirmaciones es la correcta?

i) Si $f^{-1}\left(x_{\mathrm{p}}\right)=0$ y $f^{\prime \prime}\left(x_{\mathrm{o}}\right)>0$ entonces $f$ tiene un minimo local en $x_{\mathrm{o}}$

ii) Si $f^{\prime}\left(x_{0}\right)=0$ y $f^{\prime \prime}\left(x_{0}\right)>0$ entonces $f$ tiene un máximo local en $x_{0}$

iii) Si $f^{\prime}\left(x_{0}\right)=0$ y $f^{\prime \prime}\left(x_{\mathrm{o}}\right)<0$ entonces $f$ tiene un minimo local en $x_{\mathrm{o}}$

iv) Si $f^{-1}\left(x_{0}\right)=0$ y $f^{\prime \prime}\left(x_{0}\right)=0$ entonces $f$ tiene un punto de inflexión en $x_{0}$

v) Ninguna de las anteriores.

Justifica tu respuesta.

10.- Un modelo para la propagación de un rumor dice que la razín de propagación es proporcional al producto entre el número de personas que han escuchado el rumor y el número de personas que no lo han escuchado.

En un pueblo de 1500 habitantes, Juanita recibe de su novio un anillo de brillantes y le cuenta a sus amigas más cercanas, de manera que el lunes, 8 personas conocen la noticia. El miércoles de la misma semana 80 personas se han enterado.

La ecuación diferencial que representa el problema es:

i) $\frac{d P}{d t}=k(1500-P)$

ii) $\frac{d P}{d t}=k P$

iii) $\frac{d P}{d t}=k P(1500-P)$

iv) $\frac{d P}{d t}=1500 P$

v) $\quad \frac{d P}{d t}=P(1500-P)$

Determina la solución general de la ecuación diferencial.

Figura 7 - PME - Indicadores 8 a 10

Fuente: desarrollado por los autores 